\title{
Asymptotic Giant Branch Stars Migration from the Central of the Milky Way Galaxy to our Sun's Birthplace and its Relation with Silicon Carbide Grains
}

\author{
Ahmad Hashem Abdelhadi \\ New York Institute of Technology, \\ College of Arts and Sciences, P.O. Box 840878, Amman 11184, Jordan
}

\begin{abstract}
Silicon carbide grains found in meteorites are peculiar in their age and isotopic ratios; they formed before the Sun was born and their isotopic signature indicates that they come from a different galactic region. This study aims to seek a possible paradigm for such richness and peculiarity through Monte Carlo simulation of scattering of Asymptotic Giant Branch (AGB) stars off molecular cloud. Such approach randomly generates AGB stars in regions close to the Galaxy bulge and examines possibility of migration to outer regions by scattering off molecular cloud. A successful explanation to this problem will influence how we think nuclides were formed and then distributed in the Galaxy and will shed new light unto the age and the chemical evolution of the Milky Way Galaxy. Thus, it is important that we know where do they come from and how do they end up in our backyard?
\end{abstract}

Keywords: Silicon Carbide, AGB Stars, Molecular Clouds

\section{INTRODUCTION}

Many studies (e.g., 5) have shown that the mainstream presolar Silicon Carbide (SiC) grains extracted from meteorites formed in asymptotic giant branch stars. Ion probe studies show them to have ${ }^{29} \mathrm{Si} /{ }^{28} \mathrm{Si}$ and ${ }^{30} \mathrm{Si} /{ }^{28} \mathrm{Si}$ isotopic ratios larger than those found in solar material (Fig. 1). When deviations of the ${ }^{29} \mathrm{Si} /{ }^{28} \mathrm{Si}$ from the solar $\left({ }^{29} \mathrm{Si} /{ }^{28} \mathrm{Si}\right)$, ratio are plotted against the deviations of the ${ }^{30} \mathrm{Si} /{ }^{28} \mathrm{Si}$ from the solar $\left({ }^{30} \mathrm{Si} /{ }^{28} \mathrm{Si}\right)$ ratio a best-fit slope, of the three-isotope plot, is found to be 1.34 (Hoppe et al., 1994). The essence of this research is to begin an evaluation of an explanation for the heavy-isotope excesses in the mainstream $\mathrm{SiC}$ particles within a framework of the galactic dynamics. I evaluate the idea that a percentage of AGB stars formed in the region of the Galaxy interior to solar birth, with metallicity higher than solar, change their orbital trajectory during close encounters with molecular clouds and that they migrate outward to the position of solar birth where they give out their stardust. I proceed to examine this idea and quantify such percentages. At this point one would ask; why is the isotopic richness in the presolar $\mathrm{SiC}$ grains a problem.

\subsection{The Problem of Mainstream SiC Grains}

The grain-by-grain deviations of $\mathrm{Si}$ isotopic ratios from solar composition, in parts per thousand, are shown in Fig. 1, where galactic time in a chemical evolution model is shown on the right margin (Timmes and Clayton, 1996). The obvious correlation trend seen there is called the mainstream. The problem lies in the fact that the mainstream presolar SiC grains are richer in the heavier silicon isotopes than the solar composition even though the grains must have originated in stars that formed prior to the Sun. To deliver their grains to the solar birth cloud, the donor stars clearly must have been born and evolved prior to solar birth. Homogeneous chemical evolution of these $\mathrm{Si}$ isotope ratios increase monotonically with time and simply cannot accommodate earlier stars having higher ratios. 


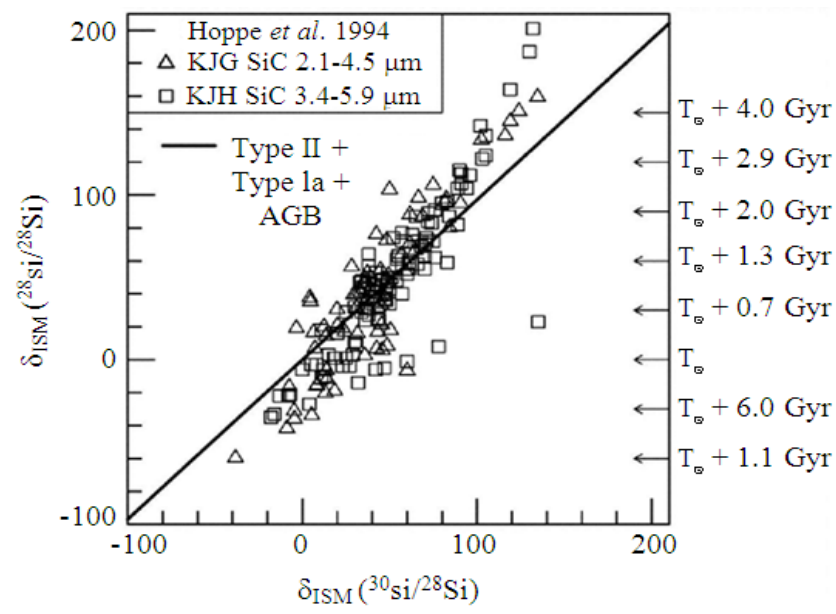

Fig. 1. Silicon isotope deviations in a three-isotope plot. Murchison SiC samples measured by Hoppe et al. (1994) are shown and have a best-fit slope of 4/3. The renormalized mean Interstellar Medium (ISM) evolution is shown as the solid line and by construction it passes through solar abundances at $\mathrm{t}=\mathrm{t}_{\odot}$. With such a construction, deviations with respect to solar abundances and with respect to interstellar abundances are the same $\delta_{\odot}=\delta_{\text {ISM }}$

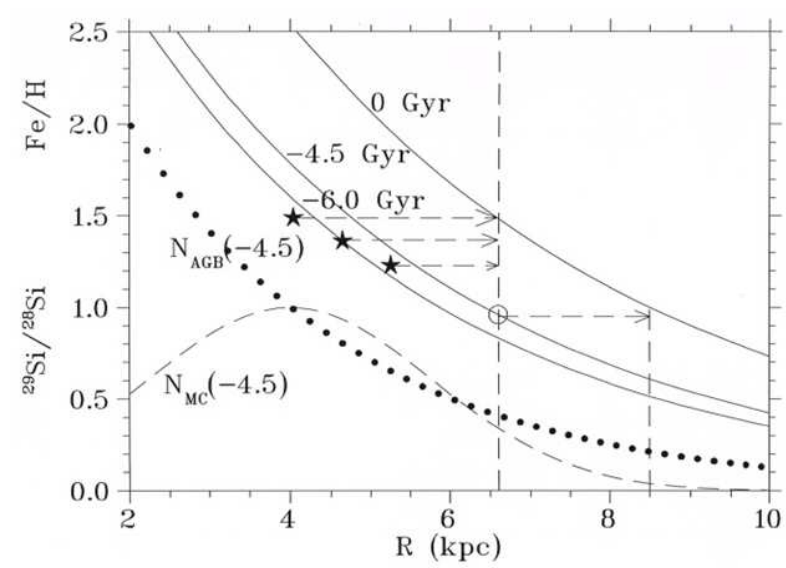

Fig. 2. Diffusion of the Sun and three stars as postulated to explain heaviness of mainstream Si. The three solid curves represent the gradients of the gas metallicity at three different times: today $(t=0)$, the time of solar birth $(t=-4.5$ Gry $)$ and $t=-6.0$ Gyr. NAGB is the number of AGB stars per unit volume and it increases toward the Galactic center as suggested schematically at $t$ $=-4.5$ Gyr. Similarly, $\mathrm{N}_{\mathrm{MC}}$ is the space density of molecular clouds and it declines toward the solar position. The sun was taken to have been born at $\mathrm{R}=6.6 \mathrm{kpc}$ (Clayton, 1997)

Therefore, one seeks reasons for the high isotopic ratios of the majority of donor stars. The assumption here is that the parent AGB stars diffuse from more central birthplaces within the Galaxy. Due to a more evolved interior position, these central birthplaces are known to have a higher metallicity. The outward diffusion can be likened to the gravity assist used in solar system exploration. AGB stars scatter by near gravitational encounters with molecular clouds. This changes the orbits of some to spend much time at larger galactic radii that those of their birth. There they give out their stardust.
A representation of the diffusion of three AGB stars is given in Fig. 2 where the three example AGB stars are shown as star symbols. They diffused from their birthplaces and arrived at the solar birth location (here taken to be $\mathrm{R}=6.6 \mathrm{kpc}$ ) at $\mathrm{t}=-4.5 \mathrm{Gyr}$ during the period of AGB loss of stardust. The analogy to planetary gravity assist applies in here but with a difference. Here, an AGB star encounters number of molecular clouds throughout its life. This in return perturbs its orbit and changes its trajectory. The accumulative effect of these encounters forces the star into a new orbit in the solar 
neighborhood where the star gives out its stardust. It's worth mentioning that this procedure is completely different from diffusion. I seek a slingshot effect where a flyby AGB star acquires a big tug at strong close encounters. It's merely gravitational scattering of AGB stars off molecular clouds.

\subsection{Asymptotic Giant Branch Stars}

The mass of a star almost determines its structure and ultimate fate. Stars begin their existence as clouds of gas that become dense enough to start contracting under the inward pull of their own gravity. The lives of stars proceed through different stages. At one point in its life a star moves onto the asymptotic giant branch stage; an extension of the red giant branch toward higher luminosities and lower effective temperature. Convection following pulses of helium burning dredge gas up from the deep interior, bringing to the surface newly formed atoms of elements such as carbon and heavier elements, which have also been neutron irradiated in the star by the s-process (Sparke and Gallagher, 2007). The surface enrichment of carbon makes it eventually more abundant that oxygen, at which point the AGB star is called a carbon star. It can then condense carbides such as $\mathrm{SiC}$ during mass loss. The stellar wind pushes polluted surface gas out into the interstellar medium. The sprocess signature for heavy elements in the silicon carbide grains confirms that they originated in AGB stars. This makes AGB stars a major source of the SiC grains. That is why they are the focus of this study.

\subsection{Gravity Assist}

In this section I will give a prelude into the Physics of gravity assist or gravity boost, as it's sometimes called, in a heliocentric (sun-centered) system. If a heavy ball rolling on a table collides with a light ball rolling in the opposite direction, its velocity will hardly change, while the lighter ball will bounce away with an increased velocity. A similar phenomenon occurs during a "gravitational collision" between a heavy planet and light spacecraft. Let $v_{i}$ and $v_{f}$ denote the spacecraft momenta in the heliocentric inertial frame just before and after the encounter. Let $\mathrm{V}$ be the velocity of a planet about the sun and $u_{i}$ and $u_{f}$ are the spacecraft velocities relative to a reference frame where the planet is at rest. One can write the Galilean transformation:

$$
\begin{aligned}
& \mathrm{v}_{\mathrm{i}}=\mathrm{u}_{\mathrm{i}}+\mathrm{V} \\
& \mathrm{v}_{\mathrm{f}}=\mathrm{u}_{\mathrm{f}}+\mathrm{V}
\end{aligned}
$$

Also the change in velocity during the encounter can be written as Equation 3:

$$
\Delta \mathrm{v} \equiv \mathrm{v}_{\mathrm{f}}-\mathrm{v}_{\mathrm{i}}
$$

which has the same value in both frames (heliocentric inertial frame and planet inertial frame) Equation 4:

$\Delta \mathrm{v}=\Delta \mathrm{u}$

For an elastic collision where the planet maintains its velocity about the sun (the planet's system of reference is practically inertial) Equation 5:

$\mathrm{u}_{\mathrm{f}}=\mathrm{u}_{\mathrm{i}}$

The spacecraft's orbit is governed by the gravitational field of the sun. However, when the spacecraft reaches the vicinity of the planet its orbit is mainly determined by the strong gravitational field of planet.

The change in squared heliocentric velocity measures the gravity assist, i.e., energy gain, in kinetic energy:

$$
\Delta \mathrm{KE}=1 / 2 \mathrm{~m}\left(\mathrm{v}_{\mathrm{f}}^{2}-\mathrm{v}_{\mathrm{i}}^{2}\right)
$$

Recall work is the change in kinetic energy. Square the two equations Equation 1 and 2:

$$
\begin{aligned}
& v_{f}^{2}=u_{f}^{2}+2 u_{f} \cdot V+V^{2} \\
& v_{i}^{2}=u_{i}^{2}+2 u_{i} \cdot V+V^{2}
\end{aligned}
$$

If we subtract these two equations, we obtain:

$$
\mathrm{v}_{\mathrm{f}}^{2}-\mathrm{v}_{\mathrm{i}}^{2}=2 \Delta \mathrm{u} . \mathrm{V}=2 \Delta \mathrm{v} . \mathrm{V}
$$

The magnitude of the heliocentric velocity increases (or decreases) depending on whether the projection of $\Delta \mathrm{u}$ on $\mathrm{V}$ is positive (or negative). If the spacecraft crosses the planet's orbit behind the planet, then $\Delta \mathrm{u}$. V will be positive and a gravitational assist will result. However, if the spacecraft crosses the planet's path in front, $\Delta \mathrm{u}$. V will be negative and the effect will be to slow down the spacecraft as seen in Fig. 3. For a given $v_{i}$ the maximum gravity boost is achieved when $\mathrm{v}_{\mathrm{f}}$ is parallel to $\mathrm{V}$. The outgoing orbit of the spacecraft around the sun is a conic section (ellipse, parabola, hyperbola) with a turning point at the location of the encounter. This new heliocentric conic section of the spacecraft orbit depends on the amount of velocity boost at the encounter. This analogy is applied to star-molecular cloud encounters. 


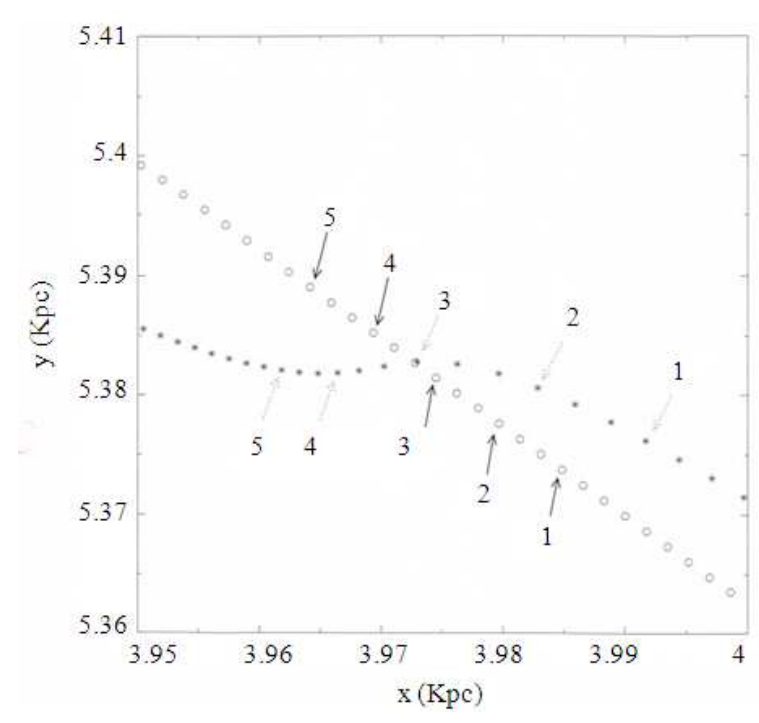

Fig. 3. A close encounter between a star and a molecular cloud. Asterisks represent the star, circles represent the molecular cloud. Both representations are equally spaced in time. Five different times are marked and they show how the molecular cloud slow down the star

\subsection{Galactic Gravitational Potential}

To calculate the orbits of stars requires a galactic mass model that defines an expression for the galactic gravitational potential. In using such a model, I neglect the forces from individual stars and consider only the largescale forces from the overall mass distribution, which is made up of thousands of millions of stars. This takes the force fields of the galaxy to be smooth, neglecting smallscale irregularities due to individual stars or larger objects like globular clusters. Hence, I use a numerically convenient axisymmetric gravitational potential and density distribution proposed by Miyamoto and Nagai (1975):

$$
\Phi(\mathrm{R}, \mathrm{z})=-\sum_{\mathrm{i}=1}^{3} \frac{\mathrm{GM}_{\mathrm{i}}}{\sqrt{\mathrm{R}^{2}+\left(\mathrm{a}_{\mathrm{i}}+\sqrt{\mathrm{z}^{2}+\mathrm{b}_{\mathrm{i}}^{2}}\right)^{2}}}
$$

where, $\mathrm{R}$ is the galactocentric radius and $\mathrm{z}$ is the height above and below the galactic mid-plane. Respectively, the three parameters a (in kpc), b (in kpc) and $\mathrm{M}$ (in $10^{10} \mathrm{M}_{\circ}$ ) are: $0.0,0.3$ and 1.8 for the bulge; 6.2, 0.4 and 17.4 for the disk; 0.0, 31.2 and 83.5 for the corona (Hartmann et al., 1990). Thus, depending on the choice of the two parameters a and $b, \Phi$ can represent the potential of anything from an infinitesimally thin disk to a spherical one. This expression is free from singularities everywhere in space and is differentiable an unlimited number of times with respect to the space coordinates. It also tends to the Newtonian potential for a point mass when $\mathrm{R}$ and $\mathrm{z}$ become large. I should also point out that I omit non-axisymmetric structures such as an inner bar, the spiral arms and local features such as Gould's Belt. However, this potential is in good agreement with observational determinations of the Galactic rotational velocity curve (Fig. 4).

The corresponding three-dimensional density distribution $\rho(R, z)$ can be derived from the Poisson's equation as (Timmes and Clayton, 1996):

$$
\rho(R, z)=\frac{1}{4 \pi} \sum_{i=1}^{3} b_{i}^{2} M_{i} \frac{a_{i} R^{2}+\left(a_{i}+3 \sqrt{z^{2}+b_{i}^{2}}\right)\left(a_{i}+\sqrt{z^{2}+b_{i}^{2}}\right)^{2}}{\left[R^{2}+\left(a_{i}+\sqrt{z^{2}+b_{i}^{2}}\right)^{2}\right]^{5 / 2}\left(z^{2}+b_{i}^{2}\right)^{3 / 2}}
$$

Which is non-negative everywhere is space.

\section{MATERIALS AND METHODS}

\subsection{Molecular Clouds}

Molecular clouds individually produce fluctuations in the gravitational field seen by nearby stars, which in return produce changes in the magnitude and direction of each stellar velocity. Thus, it becomes imperative that we establish an understanding of the physical and geometrical properties of the molecular clouds along with their distribution in the galaxy. Such understanding will point to an idealized representation of the distribution and mass ranges of the molecular clouds. Understand that this study does not seek to model the physical and geometrical properties of the molecular clouds. Such thing is beyond the scope of this study. I seek instead only a representation of the gravitational effect of molecular clouds on stellar orbits. The goal is not to study molecular clouds themselves but rather their cumulative perturbations of the stellar orbits in an azimuthally symmetric potential. The goal is to study the extent to which the radial position of stars that were born on circular orbits will be radially displaced from their circular orbits at fixed stellar evolution times. A typical star's orbit is given in Fig. $\mathbf{5}$.

Molecular clouds are detected and mapped by observing millimeter emission from a trace molecule: carbon monoxide (Pudritz, 2002). They are the dominant component of the interstellar medium in the inner half of the disk at $\mathrm{R}<0.8 \mathrm{R}_{\odot}$ with a rise between $\mathrm{R}=5$ and $\mathrm{R}=7$ $\mathrm{kpc}$ and a sharp fall toward the larger radius where there is very little emission beyond $\mathrm{R}=15 \mathrm{kpc}$. Most of the surveys (Binney and Merrifield, 1998; Pudritz, 2002; Solomon and Sanders, 1985; Ward-Thompson, 2002) suggest a median cloud mass of about $10^{5} \mathrm{M}_{\odot}$. 


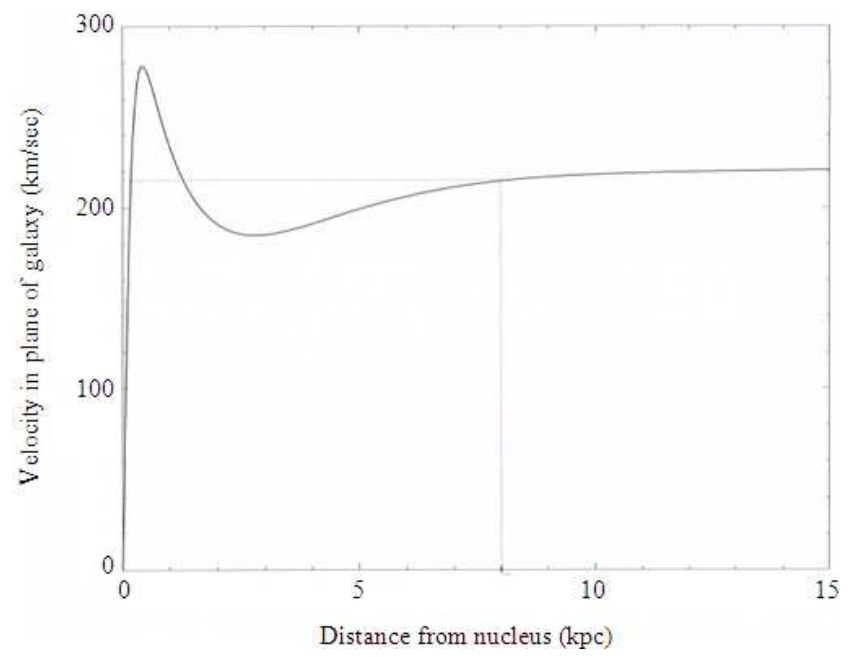

Fig. 4. A rotation curve produced using Miyamoto and Nagai (1975) potential formula. The rotation curve shows that after a rapid rise near the center of the galaxy the rotation velocity is relatively slowly varyingfor quite a large distance. The dashed line marks the Sun's position $\mathrm{R}_{\odot}$ from the galactic center at $8.5 \mathrm{kpc}$ and the Sun's rotational velocity $217 \mathrm{~km} \mathrm{sec}^{-1}$. Current observations of the Sun's rotational velocity is $220 \pm 5 \mathrm{~km} \mathrm{sec}^{-1}$ (Elmegreen, 1998)

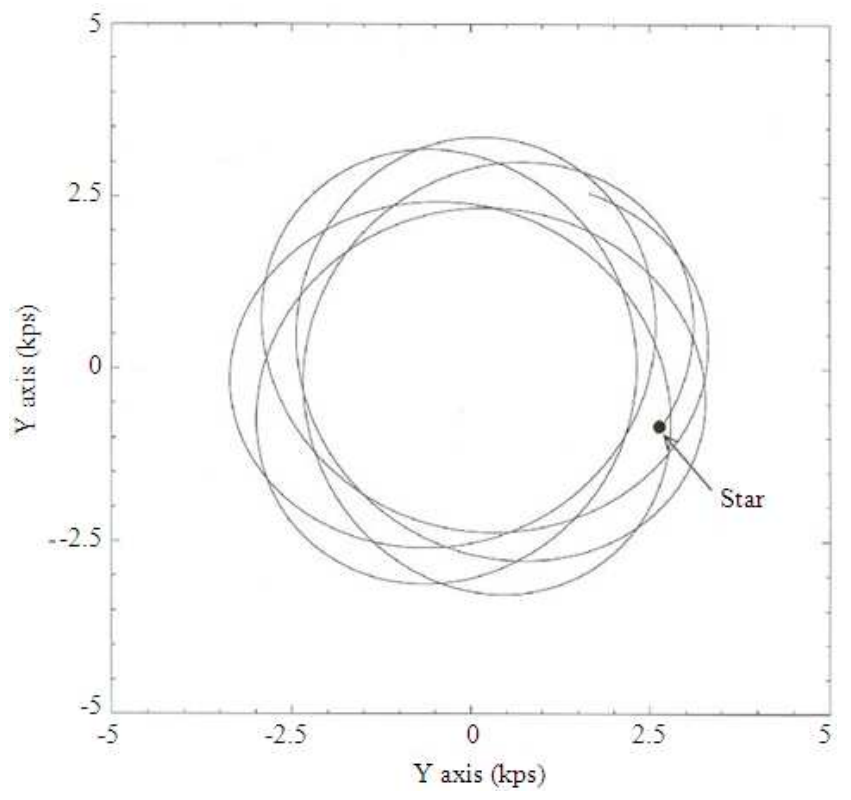

Fig. 5. A face-on galactic plane orbit that forms a rosette. The star has an initial circular velocity $196.2 \mathrm{~km} \mathrm{sec}^{-1}$ and lived for 0.5 billion years

The age of a Molecular Cloud ranges between $10^{5}$ years and several times $10^{8}$ years, depending on the cloud's size and mass, among other things.

The axisymmetric distribution of molecular clouds in the galactic disk shows that most of the molecular clouds in our galaxy are within a region between 4 and $8 \mathrm{kpc}$ from the galactic center. However, the radial distribution is not simply determined. Thus, no radial distribution or mass range for the molecular clouds will be easy or exact. But the objective of this study is to tackle a physics problem in which a star falls into the gravitational field of a neighboring molecular cloud and 
is forced to change its orbital trajectory. It is not my intention to model neither the molecular clouds nor their radial distribution. Moreover, my problem concerns the effect of the molecular clouds on the motion of stars through an otherwise azimuthally symmetric potential, therefore, I can utilize a simple approximation of their masses and the radial distribution for that purpose. A simple model cannot take into account the geometrical shape of the molecular clouds. To a first approximation I can take the molecular clouds to be spherical.

Although the masses of the molecular clouds could be assigned for each cloud randomly, I have chosen instead to use one fixed mass for all clouds. The orbital problem is computationally intensive. Given the fact that most of the surveys suggest a median mass about $10^{5} \mathrm{M}_{\odot}$, I assume that mass for all.

I employ a Gaussian distribution that randomly generates positions for the molecular clouds along the galactic mid plane. The transformation I use for the Gaussian deviations is (Press et al., 1992):

$$
\mathrm{R}=\sqrt{-2 \ln \mathrm{a}_{1}} \cos 2 \pi \mathrm{a}_{2}
$$

where, $\mathrm{R}$ is the galactocentric radius, $a_{1}$ and $a_{2}$ are two uniform deviates on $(0,1)$. The Gaussian distribution presented here is not fully axisymmetric nor smooth because $10^{4}$ clouds are distributed randomly; meaning strings of high dense areas of molecular clouds are instituted and placed randomly to account for the observed fluctuation in the radial distribution. A more realistic distribution is something to be considered in any future work.

To compute the scattering of a star by a spherical cloud two simple approaches can simplify the calculations. A softening parameter that alters the density configuration of the cloud. Or alternatively, one might assume the cloud to have constant density. Both approaches eliminate unrealistically large forces near the cloud center. The softened potential and the constant density are simply models with different density distribution.

\section{RESULTS}

Before I introduce my results I explain how I obtained them. The code I have used follows the orbits of stars and molecular clouds by integrating numerically the equation of motion for the stars. The massive clouds remain on their circular orbit. This is done by solving the second order differential equation; Newton's second.
A numerical method called Bulirsch-Stoer (Press et al., 1992) algorithm with adaptive time step was used for this purpose. Bulirsch-Stoer method is very efficient when high-accuracy solutions for ordinary differential equations are desired. The idea is to start with an initial value and calculate the value at the end of an interval many times with successively finer steps and then fit a polynomial or a rational function to extrapolate to the magical limit of an infinitely small step size. A single Bulisrsch-Stoer step takes a stellar coordinate from $x$ to $\mathrm{x}+\mathrm{H}$, where $\mathrm{H}$ is supposed to be quite a large distance. The single step usually consists of many substeps, each of size $h=H / n$, of the so called modified midpoint method, which are extrapolated to a mythical zero step size $(\mathrm{h}=0)$ using a rational function. Both the consecutive step sizes and the number of substeps are determined as functions for a predefined accuracy limit. This way the code doesn't keep on subdividing $\mathrm{H}$ infinitely. The sequence of separate attempts to cross the interval $\mathrm{H}$ is made with increasing values of the number substeps n. For each step it's not known in advance how far up this sequence one must go. After each successive $\mathrm{n}$ is tried, a polynomial extrapolation is attempted. The extrapolation returns both extrapolated values and error estimates. If the errors are not satisfactory, the code goes to a higher n. If they are satisfactory, it goes on to the next step.

A typical calculation is set up to trace $10^{4}$ molecular clouds and $10^{3}$ stars for two billion years. I place the molecular clouds randomly on circular orbits in the galactic mid plane. The clouds do not have peculiar velocities thus their motions do carry them in the $\mathrm{z}$ direction and their circular orbits are not perturbed, i.e., they do not oscillate radially nor azimuthally. Each cloud has mass $10^{5} \mathrm{M}_{\odot}$ and $10 \mathrm{pc}$ radius. Since each molecular cloud is more massive than the star; a gravitational force from a nearby star on the molecular cloud is going to be small and thus neglected. To a first approximation the molecular clouds retain their circular orbits and constant angular momentum. The stars are initially distributed randomly in radial zones. A zone is a ring with $500 \mathrm{pc}$ width; for example, the region between $\mathrm{R}=4.0 \mathrm{kpc}$ and $\mathrm{R}=4.5 \mathrm{kpc}$ is one zone. Each zone has $10^{3}$ stars positioned randomly in $\mathrm{R}$ and in its azimuthal angle in the galactic mid plane. Each star is given a randomly generated peculiar velocity between 10 and $20 \mathrm{~km} \mathrm{sec}^{-1}$. The direction of the peculiar velocity is also randomly chosen in $\theta$ and $\phi$, where $\theta$ and $\phi$ are the known spherical coordinates. Added to the peculiar velocity is the proper circular velocity deduced from Miyamoto and Nagai potential. With these initial conditions the system evolves for two billion years. Both molecular clouds and stars maintain their shapes and masses throughout collisions and calculations. 
Recording the positions for the molecular clouds and the stars for each zone at each time step can be cumbersome. Therefore, only points at $\mathrm{t}=10^{9}, 1.25 \times 10^{9}$, $1.5 \times 10^{9}, 1.75 \times 10^{9}$ and $2 \times 10^{9}$ years are recorded. These are interesting ages for AGB stars. In order not to miss on any maximum displacement that a star could have accrued between these times, I also record the maximum displacement a star reaches during its lifespan. Upon comparing these maximum values with initial conditions for all stars, one realizes that some stars went deeper toward the centre of the galaxy which made their initial conditions their maxima.

The total force F (per unit mass) on a star is the sum of the galactic gravitational force derived from Miyamoto and Nagai potential and molecular-cloud gravitational forces. A star's trajectory is mainly governed by the 'nearby' molecular clouds' gravitational field. The lengthy nature of this type of calculations suggests another approximation: only forces from nearby molecular clouds are included in the total F in Newton's scond law. Nearby molecular clouds defined as ones found within a cube of $500 \mathrm{pc}$ from a star at its center. Molecular clouds outside the cube are not included specifically in the total force at that particular point of time. The distant clouds are assumed part of the azimuthal galactic potential. This approach speeds up the calculations noticeably, because the distances between a star and the molecular clouds are already computed to select the near ones. All that is needed is an "if" statement to sort out nearby molecular clouds.

\section{DISCUSSION}

As I described earlier, the stars initial and final conditions are recorded. This is converted for display to a distribution of their changes in radial position at each time. The percentage of the number of stars that have moved radially by $\Delta \mathrm{r}$ at these different ages is also recorded. A histogram for each calculation is produced and it shows the actual number of stars, called frequency, binned at the aforementioned ages (Fig. 6 and 7). The centered-at-zero bell shape in these figures suggests that most of the stars experience a small perturbation in their orbits. The stars are less likely to deviate largely from their initial circular orbits. Close to $40 \%$ of the stars suffered inward migration. Only a small fraction of AGB stars $\sim 1 \%$ migrated to larger radii. Wielen et al. (1996) found that the Sun was born at galactocentric radius $\mathrm{R}=$ $6.6 \mathrm{kpc}$ and has in $4.5 \mathrm{Gyr}$ diffused outwards to its present location at $\mathrm{R}=8.5 \mathrm{kpc}$. My calculations found in two billion years a percentage of $0.4 \%$ stars born between 4.0-4.5 kpc migrate outward to $6.0-6.5 \mathrm{kpc}$ zone where they give their AGB stardust to a presolar cloud. This result, in particular, is very interesting because Sellwood and Binney (2002) studied the scattering of stars by spiral waves and concluded that the grains must have migrated from $\mathrm{R} \sim 4 \mathrm{kpc}$ to $\sim 6 \mathrm{kpc}$ where they were incorporated into a presolar cloud.

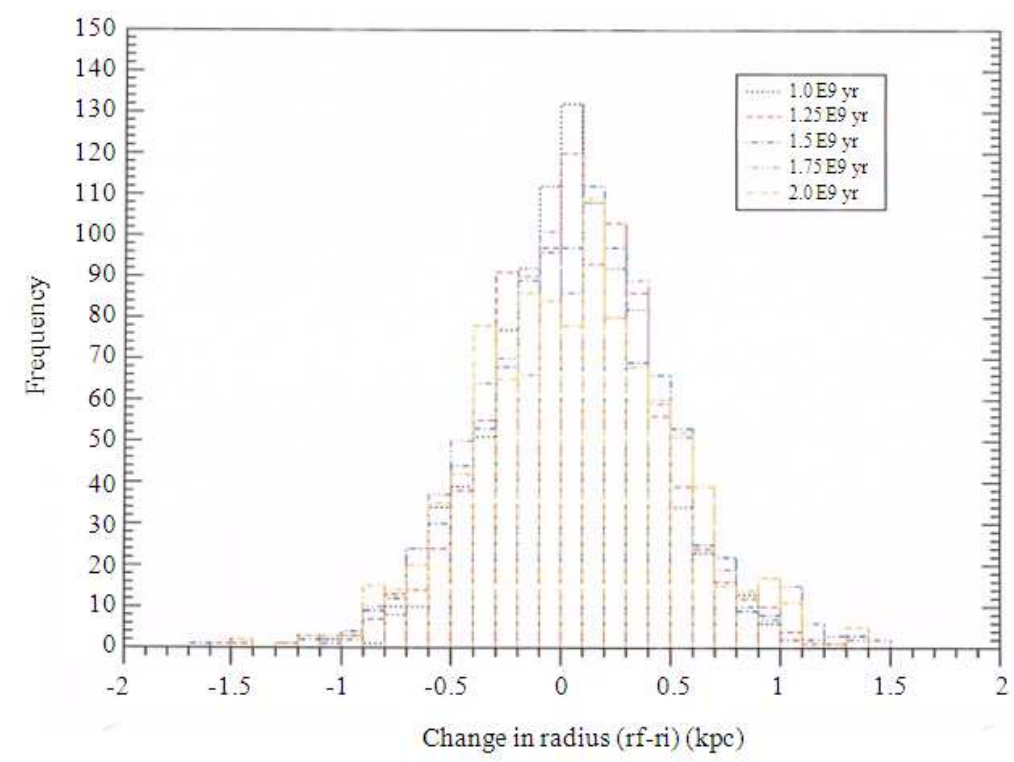

Fig. 6. Region 3.5-4.0 kpc. The 1000 stars binned at different ages. The x-axis is the difference between the initial galactocentric radius and the final radius for each star 


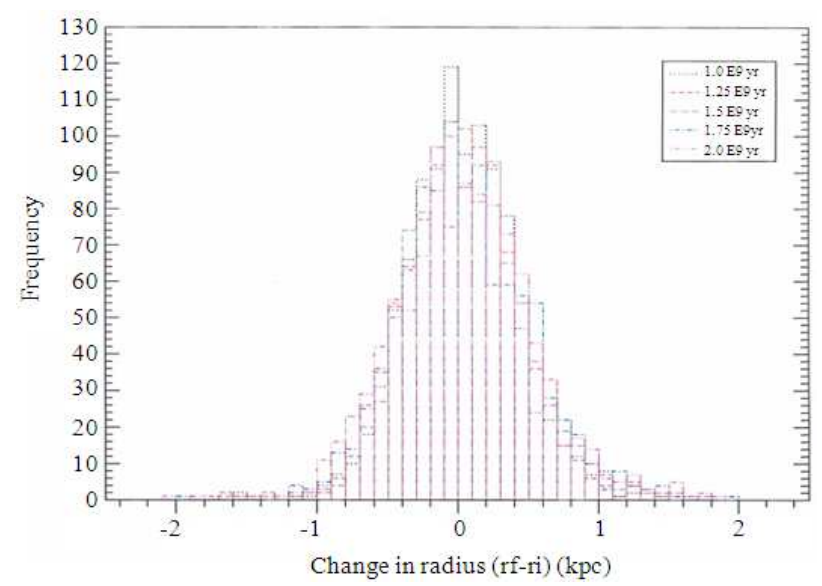

Fig. 7. Region 3.5-4.0 kpc. The 1000 stars binned at different ages. The x-axis is the difference between the initial galactocentric radius and the final radius for each star

\section{CONCLUSION}

The results show close encounters between AGB stars and molecular clouds are apparently too infrequent to contribute primarily to the problem of silicon isotopic rations in mainstream $\mathrm{SiC}$ grains. Migration of AGB stars from more central regions in the galaxy due to scattering by molecular clouds is deemed unlikely. The percentages the calculations have produced are small and cannot account for the observed richness in the heavier silicon isotopes when compared to solar values.

One hoped to see a larger fraction of stars scattered from their initial almost circular orbits to larger ones. I have shown only a few stars do that.

\section{ACKNOWLEDGEMENT}

The NASA Origin of Solar Systems Program has supported this study.

\section{REFERENCES}

Binney, J. and M. Merrifield, 1998. Galactic Astronomy. 1st Edn., Princeton University Press, Princeton, ISBN-10: 0691025657, pp: 796.

Clayton, D.D., 1997. Placing the sun and mainstream $\mathrm{SiC}$ particles in galactic chemodynamic evolution. Astrophys. J. Lett., 484: L67-L70. DOI: $10.1086 / 310768$

Elmegreen, D.M., 1998. Galaxies and Galactic Structure. 1st Edn., Prentice Hall, New Jersey, ISBN-10: 0137792328, pp: 306.

Hartmann, D., S.E. Woosley and R.I. Epstein, 1990. Galactic neutron stars and gamma-ray bursts. Astrophys. J., 348: 625-633.
Hoppe, P., S. Amari, E. Zinner, T. Ireland and R.S. Lewis, 1994. Carbon, nitrogen, magnesium, silicon and titanium isotopic compositions of single interstellar silicon carbide grains from the Murchison carbonaceous chondrite. Astrophys. J., 430: 870-890.

Miyamoto, M. and R. Nagai, 1975. Three-dimensional models for the distribution of mass in galaxies. Publ. Astron. Soc. Japan, 27: 533-543.

Press, W.H., B.P. Flannery, S.A. Teukolsky and W.T. Vetterling, 1992. Numerical Recipes in Fortran. 2nd Edn., Cambridge University Press, Cambridge, ISBN-10: 052143064x, pp: 992.

Pudritz, R.E., 2002. Clustered star formation and the origin of stellar masses. Science, 295: 68-75. DOI: 10.1126/science. 1068298 .

Sellwood, J.A. and J. Binney, 2002. Radial mixing in galactic discs. MNRAS, 336: 785-796. DOI: $10.1046 / \mathrm{j} .1365-8711.2002 .05806 . x$

Solomon, P.M. and D.B. Sanders, 1985. Star formation in a galactic context-the location and properties of molecular clouds. Protostars Planets II, A86-12626: 59-80.

Sparke, L.S. and J.S. Gallagher, 2007. Galaxies in the Universe: An Introduction. 2nd Edn., Cambridge University Press, Cambridge, ISBN-10: 1139462385, pp: 431.

Timmes, F.X. and D.D. Clayton, 1996. Galactic evolution of silicon isotopes: Application to presolar $\mathrm{SiC}$ grains from meteorites. Astrophys. J., 472: 723. DOI: $10.1086 / 178102$

Ward-Thompson, D., 2002. Isolated star formation: From cloud formation to core collapse. Science, 295: 76-81. PMID: 11778038

Wielen, R., B. Fuchs and C. Dettbarn, 1996. On the birthplace of the sun and the places of formation of other nearby stars. Astronomy Astrophys., 314: 438-447. 